\title{
Modélisation de signaux sonores par transformées temps-échelle et temps-fréquence
}

\author{
G. COURBEBAISSE, B. ESCUDIÉ, Ph. GUILLEMAIN* et R. KRONLAND-MARTINET*
}

ICPI-CPE, Laboratoire de Traitement du Signal (LTS), 31 Place Bellecour, 69288 Lyon cedex, France ${ }^{*}$ CNRS, Laboratoire de Mécanique et d'Acoustique (LMA), 31 Chemin Joseph Aiguier, 13402 Marseille cedex 09, France

Résumé: Les représentations énergétiques en temps et échelle, ou en temps et fréquence distribuent l'information contenue dans les modulations du signal en fonction de ces variables. Pour les signaux à modulation linéaire d'amplitude et sinusoïdale en fréquence, ces procédés mettent en évidence le retard de groupe et la fréquence instantanée. Trois procédés d'étude, scalogramme (ondelette en module carré), représentation de Wigner-Ville et représentation de Unterberger, version passive, permettent d'extraire les paramètres de modulation de signaux rencontrés en acoustique musicale.

Abstract: Energetic time-scale representations or time-frequency representations deal with data related to the signal modulations, with a dependance on the upper variables. For linear amplitude modulations and sinusoidal frequency ones, these tools point out the group delay and the instantaneous frequency. Extraction of modulation parameters on music acoustical signals, is provided by the scalogram (square modulus of the wavelet transform), the Wigner-Ville time-frequency representation and the passive Unterberger time-scale representation.

\section{INTRODUCTION.}

Depuis l'introduction du procédé d'analyse par ondelettes, diverses représentations en temps et échelle furent proposées [1], [2], [3]. Elles tiennent compte des transformations de décalage temporel et de changement d'échelle [2]. Leur lien avec la représentation de Wigner-Ville passe par une transformation affine [2]. La comparaison des résultats obtenus avec différentes représentations, sous des contraintes diverses, permet de juger leur aptitude à extraire les paramètres de modulation d'un signal.

\section{REPRESENTATIONS ETUDIEES DANS LE CAS PRESENT.}

Etant donné un signal certain $S$ d'énergie finie $\left(S \in L^{2}\right)$, de transformée de Fourier : $s(v)=S(t)$, on définit [1], [2]:

$$
\mathbf{T}_{s}(t, \eta)=\sqrt{\eta} \int_{R} z(u) G^{*}(\eta(u-t)) d u
$$

Ts est la représentation en ondelette de $S$, calculée avec l'ondelette analysante $G(t)$ et $Z(t)$ le signal analytique associé à $\mathrm{S}(\mathrm{t})$. On appelle scalogramme la quantité suivante:

$$
\left|\tau_{s}(t, \eta)\right|^{2}=\eta\left|\int_{R} z(u) G^{*}(\eta(u-t)) d u\right|^{2}
$$

G est en général de forme admissible, soit: $g(0)=\int_{R} G(t) d t=0$

pour obtenir une reconstruction aisée de $S$ à partir de $\mathcal{T}_{\mathbf{S}}$ [1]. 
Ayant défini la représentation de Wigner-Ville par:

$$
W_{z}(t, v)=\int_{R} Z\left(t+\frac{\tau}{2}\right) \cdot Z^{*}\left(t-\frac{\tau}{2}\right) \cdot e^{-2 i \pi \nu \tau} d \tau
$$

on envisage dans la classe Wigner affine la distribution dite Grossmann-Unterberger passive[3]:

$$
B_{z}(t, \eta)=\eta v_{M} \int_{R^{+}} \frac{1}{v}, z(\eta v) \cdot z^{*}\left(\eta \frac{v_{M}^{2}}{v}\right) e^{2 i \pi \eta t\left(v-\frac{v_{M}^{2}}{v}\right)} d v
$$

Dans les applications envisagées on utilise la propriété d'extraction de la fréquence instantanée, ou celle de retard de groupe associé, par ces procédés[4].

$$
v_{i}(t)=\frac{1}{2 \pi} \frac{d \Phi}{d t}, Z(t)=|Z| e^{i \Phi} ; z(v)=|z| e^{i \varphi}, \tau_{g}(v)=-\frac{1}{2 \pi} \frac{d \varphi}{d v}
$$

\section{TYPE DE SIGNAL ANALYSE.}

Dans l'étude des signaux acoustiques vocaux ou musicaux, il apparaît des effets de vibrato manifestés par l'étude des modulations de fréquence sinusoïdale, de fréquence F.[5] Ceci conduit à l'expression analytique:

$$
S(t)=A(t) \sin \left(2 \pi v_{0} t+J(t) \sin (2 \pi F t)\right)
$$

où $v_{0}$ et $F$ sont des fréquences fixes et $\mathrm{J}(\mathrm{t})$ une fonction continue représentant l'évolution de la modulation de fréquence sinusoïlale, de fréquence $F$. Nous considérons ici $J_{1}(t)$ et $J_{2}(t)$ :

$$
J_{1}(t)=a t=\frac{t}{T}, J_{2}(t)=1-\left|\frac{t-T / 2}{T / 2}\right|
$$

Dans les deux cas, les paramètres de $S(t)$ sont les suivants:- $v_{0 / F}=24, v_{0} / F=10$, avec $v_{0}=500 \mathrm{~Hz}$, $\mathrm{T}=255 \mathrm{~ms}$. La figure 1 représente la densité spectrale d'énergie des signaux étudiés.
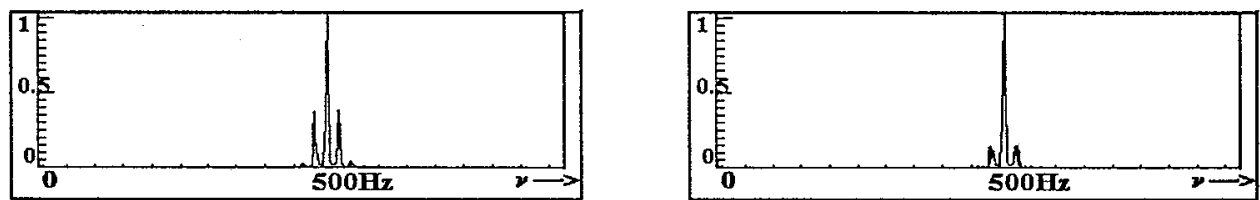

figure 1 Densité spectrale d'énergie de $S 1(t, J 1(t))$ et de $S 2(t, J 2(t))$

\section{ANALYSE PAR ONDELETTE.}

Les figures 2 et 3 donnent le module de la transformée en ondelette pour les deux signaux étudiés $\left(v_{0 / F}=24, v_{0} / F=10\right)$ en utilisant l'ondelette de Morlet $\left(\omega_{0}=5.3\right)$. Dans chaque cas la fréquence instantanée a été extraite en calculant l'arête de l'image temps-fréquence associée., les signaux et l'ondelette étant asymptotiques...[6]. Dans le cas de $S(t, J 1), I(t)$ est mesuré sans biais sur la représentation tempsfréquence. Dans le cas de $S(t, J 2)$, il en est autrement, le biais sur $I(t)$ atteint $7 \mathrm{~Hz}$ pour $t=T / 2$. Dans les deux cas, la loi de modulation d'amplitude est restituée.

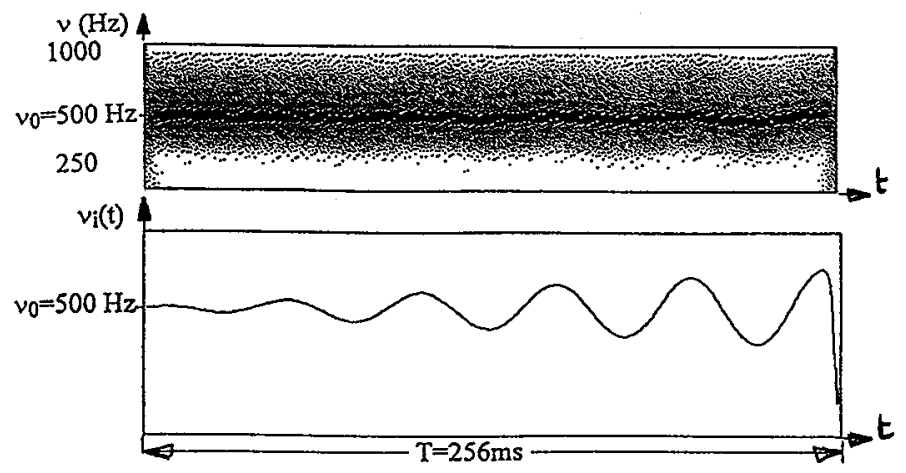

figure 2 Module de la transformée en Ondelette et fréquence instantanée de $S(t, J 1)$ 


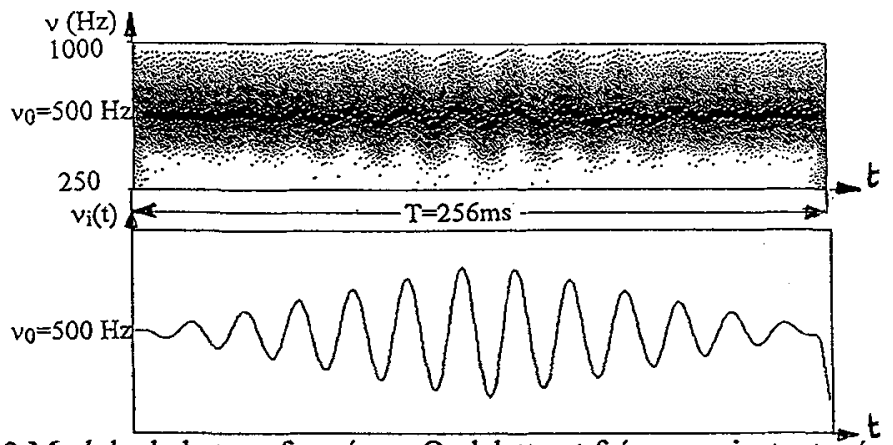

figure 3 Module de la transformée en Ondelette et fréquence instantanée de $S(t, J 2)$

\section{ANALYSE PAR REPRESENTATIONS TEMPS-FREQUENCE ET TEMPS-ECHELLE.}

5.1.Représentation de Wigner-Ville (4).

Les figures 4 et 5 fournissent le résultat pour $v_{0 / F}=24, J_{1}(t)$ (cf relation 8 ) et $v_{0} / F=10, J_{2}(t)$ (cf 8$)$. On observe une forte localisation de l'énergie dans le plan $(t, v)$ au voisinage de la courbe $v=v_{i}(t)$. Ceci est dû au caractère asymptotique du signal lié au paramètre $v_{0} T \approx 128$ pour le cas $v_{0 / F}=24, J_{1}(t)$ (cf relation 8). Le calcul du moment du premier ordre fournit aisément $v_{i}(t)$ :

$$
v_{i}(t)=\frac{\int_{R} v \cdot W_{Z}(t, v) d v}{\int_{R} W_{Z}(t, v) d v}
$$

ce qui conduit bien à: $\mathrm{F}=20.8 \mathrm{~Hz}$ observée au point $\mathrm{t}=\mathrm{T}=255 \mathrm{~ms}$. La figure 5 fournit une représentation moins lisible du signal liée au fait que $v_{0} \mathrm{~T}=128$ avec $v_{0} / \mathrm{F}=10$. Le calcul du moment du premier ordre conduit à $\mathrm{F}=50 \mathrm{~Hz}$, observé au point $\mathrm{t}=\mathrm{T} / 2$. L'estimation de l'amplitude $\mathrm{A}(\mathrm{t})$ et de la densité spectrale $\gamma_{\mathrm{z}}=|\mathrm{z}(v)|^{2}$ est directe à l'aide des moments d'ordre zéro (cf figure 5).
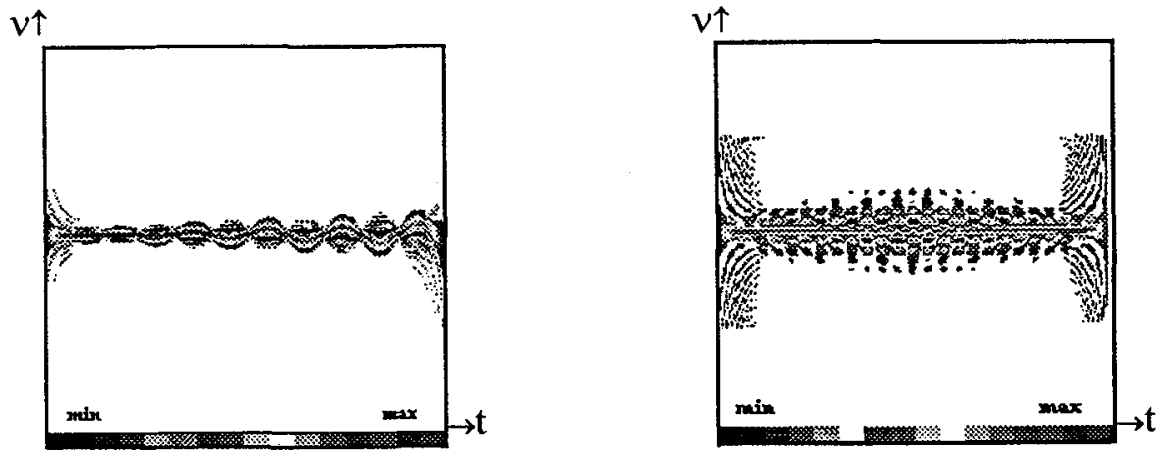

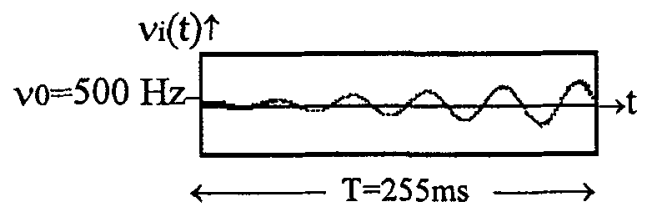

figure 4 Wigner-Ville de $S\left(t, J_{1}(t)\right)$ et fréquence instantanée associée

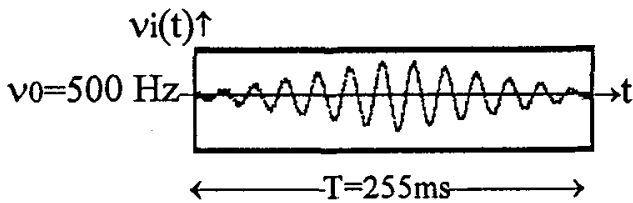

figure 5 Wigner-Ville de $S\left(t, J_{2}(t)\right)$ et fréquence instantanée associée

\subsection{Représentation temps-échelle Unterberger passive (5).}

La figure 6 a trait au signal correspondant à $v_{0} / F=24, J 1(t)$. La structure des termes d'interférence conforme aux prédictions [7], n'empèche pas l'observation d'un lieu central lié au retard de groupe $\tau_{\mathrm{g}}(v)$. 
L'estimation du moment du premier ordre permet d'extraire $\tau_{g}\left(\eta v_{M}\right), v=\eta v_{M}$, où $v_{M}$ est la fréquence de référence telle que $v_{M}=v_{1} . v_{2}$, avec $v_{1}=(4 / 10) v_{0}, v_{2}=1,6 v_{0}, v_{M}=400 \mathrm{~Hz}$

Le choix du support du facteur d'échelle est arbitraire dans notre cas [8]. Il est défini par:

$$
\sqrt{\frac{v_{1}}{v_{2}}}=\frac{1}{2} \leq \eta \leq 2=\sqrt{\frac{v_{2}}{v_{1}}}
$$

Malgré la présence des interférences, le calcul de $\tau_{g}$ fournit au point $t=T / 2$ le paramètre $F / 2=10,4 \mathrm{~Hz}$.
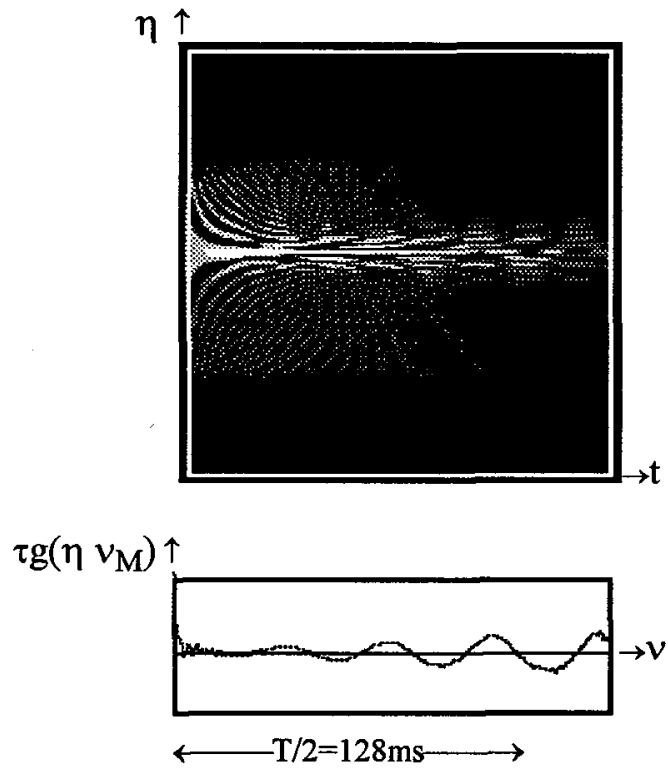

figure 6 Unterberger passive de $S\left(t, \mathrm{~J}_{1}(\mathrm{t})\right)$ et retard de groupe associé

\section{CONCLUSION.}

Les résultats montrent que l'analyse par ondelette permet d'extraire aisément $A(t)$ loi de modulation d'amplitude. Les représentations en temps et fréquence ou temps et échelle conduisent à démoduler le signal et à extraire les paramètres caractéristiques $v_{0}, \mathrm{~F}$. Elles conduisent aussi à identifier la densité spectrale et la loi de modulation d'amplitude.

\section{Références.}

[1] Morlet J.and Grossmann A., Decomposition of Hardy functions into square integrable wavelets of constant shape, S.I.A.M., Journ. Math. Anal., Vol. 15 N4, pp. 723-736, 1984.

[2] Flandrin P., Sur une classe générale d'extensions affines de la distribution de Wigner-Ville, Coll. Nat. Trait. Signal 13ième GRETSI, Juan les Pins, tome 1, pp. 17-20, 1991.

[3] Kronland R. et al., Traitement des signaux acoustiques asymptotiques par représentation tempséchelle, 2ième coll. S.F.A. Arcachon, J. Phys. IV, coll. c1 vol.2, pp. 1117-1120, 1992.

[4] Flandrin P., Temps-fréquence, Hermès Ed. pp. 117-130 et pp.140-148, 1993.

[5] Brown F., Musique par ordinateur, Que sais-je? N²011, P.U.F. Ed., Paris, 1982.

[6] Delprat N.et al., Asymptotic wavelet and Gabor analysis: extraction of modulation frequency, IEEE Transaction on Information Theory, special issue on wavelet transform and multiresolution signal analysis, Vol.38 N², pp644-664, 1992.

[7] Goncalvès $P$. et Flandrin P., Sur la localisation et la géométrie des distributions affines, Coll. Nat. Trait. Signal 14ième GRETSI, Juan les Pins, tome 1, pp. 355-358, 1993.

[8] Courbebaisse G.., Transformée Bilinéaire temps-échelle des signaux asymptotiques d'énergie finie, Coll. Nat. Trait. Signal 14ième GRETSI, Juan les Pins, tome 1, pp. 41-44, 1993. 